Section Editor

Mitchell S.V. Elkind,

MD, MS

Benjamin Tolchin, MD, MS

Joshua Z. Willey, MD, MS

Kenneth Prager, MD

Correspondence to

Dr. Tolchin:

tolchin@post.harvard.edu

Education Research:

\title{
A case-based bioethics curriculum for neurology residents
}

\section{ABSTRACT}

Background: In 2012, the American Academy of Neurology (AAN) updated and expanded its ethics curriculum into Practical Ethics in Clinical Neurology, a case-based ethics curriculum for neurologists.

Methods: We piloted a case-based bioethics curriculum for neurology residents using the framework and topics recommended by the AAN, matched to clinical cases drawn from Columbia's neurologic services. Our primary outcome was residents' ability to analyze and manage ethically complex cases as measured on precurriculum and postcurriculum multiple-choice quizzes. Secondary outcomes included precurriculum and postcurriculum self-assessed comfort in discussing and managing ethically complex cases, as well as attendance at ethics discussion sessions as compared to attendance at other didactic sessions.

Results: Resident performance on quizzes improved from $75.8 \%$ to $86.7 \%$ ( $p=0.02$ ). Comfort in discussing ethically complex cases improved from 6.4 to 7.4 on a 10-point scale ( $p=0.03$ ). Comfort in managing such cases trended toward improvement but did not reach statistical significance. Attendance was significantly better at ethics discussions (73.5\%) than at other didactic sessions $(61.7 \%, p=0.04)$.

Conclusion: Our formal case-based ethics curriculum for neurology residents, based on core topics drawn from the AAN's published curricula, was successfully piloted. Our study showed a statistically significant improvement in residents' ability to analyze and manage ethically complex cases as measured by multiple-choice tests and self-assessments. Neurology ${ }^{\circledR} 2015 ; 84$ :e91-e93

\section{GLOSSARY}

AAN = American Academy of Neurology; ACGME = Accreditation Council for Graduate Medical Education

Clinicians and medical educators from numerous specialties including neurology have noted an increasing quantity and complexity of bioethical dilemmas and have advocated formal ethical training as part of all residency curricula. ${ }^{1,2}$ The goal of this training is twofold:

1. To supply the knowledge and cognitive skills necessary for analyzing and making ethical decisions in complex clinical environments

2. To promote the specific attitudes and values deemed necessary to the moral development of the health care professional—a process of "professionalization" or "moral enculturation"3,4

In 1996, the Accreditation Council for Graduate Medical Education (ACGME) mandated that every residency curriculum must include ethics education, for the specific purpose of promoting the ACGME core competency of Professionalism. ${ }^{5}$

In 2000, the American Academy of Neurology's (AAN's) Ethics, Law, and Humanities Committee published the first pilot ethics curriculum specifically designed for neurology residents. ${ }^{6}$ One study showed this older curriculum to be effective in improving residents' knowledge of bioethics. ${ }^{7}$ In 2012, the AAN updated and expanded this pilot curriculum into Practical Ethics in Clinical Neurology: a case-based ethics curriculum for residents, fellows, and practicing neurologists. ${ }^{8}$ This is the curriculum currently recommended by the AAN for compliance with the ACGME's requirement for ethics education in residency.

Prior to July 2011, the Columbia University neurology residency program lacked a formal ethics curriculum. Our objective therefore was to demonstrate that a formal case-based bioethics curriculum, specifically

From New York-Presbyterian Hospital/Columbia University Medical Center, New York, NY

Go to Neurology.org for full disclosures. Funding information and disclosures deemed relevant by the authors, if any, are provided at the end of the article. 
employing the current topics and framework laid out by the AAN, could improve residents' ability and selfassessed comfort in discussing and managing ethically complex cases. Our hypothesis was that residents' skill and comfort would improve over the course of the curriculum.

METHODS Study population. The study involved all 31 residents in Columbia University Medical Center's neurology department. Of these, 24 residents completed precurriculum and postcurriculum quizzes and surveys, with 7 others not completing both evaluations.

Intervention. The authors first created a list of key topics from the AAN's 2000 and expanded 2012 ethics curricula, focusing on those topics that residents found most challenging and with which they most frequently grappled. ${ }^{6,8}$ Topics were drawn from the overarching categories of professionalism, clinical decisionmaking, death and dying, and special topics in neuroethics. These topics were then matched to cases from Columbia University's inpatient and outpatient neurologic services. This was in keeping with the recommendation of many medical educators and ethicists, who assert that trainees are most actively engaged in case-based discussions, and particularly when these involve real cases in which the trainees have actually participated. ${ }^{1}$ For example, the case of an elderly woman newly diagnosed with glioblastoma multiforme, whose family asked that she not be informed of the diagnosis, was used to address the issues of confidentiality and truth-telling (key topics within the domain of professionalism). The case of a man with amyotrophic lateral sclerosis who publically announced his intention to commit suicide rather than accept tracheotomy was used to discuss the topics of advanced directives (a key topic related to clinical decision-making), physicianassisted suicide, and palliative care (key topics related to death and dying).

The curriculum began with simulation sessions utilizing professional actors provided by Columbia University Medical Center's Simulation Center. All postgraduate year 2 residents completed a short didactic session on the 6-step protocol setting, perception, invitation, knowledge, emotions, summary (SPIKES) for delivering bad news to patients. ${ }^{9}$ The residents then each practiced delivering a poor diagnosis to a simulated patient/actor in an ethically complex situation (e.g., delivering news of likely brain death to a family that does not accept brain death for religious reasons). They then watched their coresidents providing poor diagnoses, and engaged in mutual feedback with the guidance of senior simulation center and neurology department attendings.

Following the introductory simulation sessions, all bioethics discussions occurred during mandatory 1 hour noon conferences and were generally formatted along the following guidelines: a resident or attending would briefly present a clinical case; whenever possible, one that he or she had actually managed. The case presentation would then be followed by a discussion facilitated by at least 2 discussion leaders. These discussion leaders were senior attending physicians including at least one neurologist and at least one member of the hospital ethics committee. The ethics committee members were typically not neurologists, and were able to offer insights from specialties such as critical care medicine, internal medicine, psychiatry, and palliative care. The authors met with the discussion leaders and presenting residents before each case discussion to determine which specific topics would be addressed in connection with the presented case.
Outcomes. Our primary outcome was residents' ability to analyze and manage ethically complex cases as measured before and after the bioethics curriculum, using a quiz of multiple choice questions regarding the proper management of ethically complex cases. The quiz questions and answers were previously vetted by members of the neurology department, the hospital ethics committee, and the division of palliative care. Secondary outcomes included the residents' self-assessed comfort in discussing and managing ethically complex cases, measured on surveys before and after the ethics curriculum using a 1-10 scale.

Another secondary outcome was resident attendance at 8 of the monthly bioethics discussion sessions, compared to resident attendance at the 8 noon conferences immediately preceding those bioethics discussions.

The study was approved by Columbia University Medical Center's institutional review board.

Statistical analysis. Precurriculum and postcurriculum quiz scores were compared for each resident as matched pairs using the Wilcoxon signed-rank test. Precurriculum and postcurriculum self-assessed comfort in discussing ethically complex cases was similarly compared, as was precurriculum and postcurriculum self-assessed comfort in managing ethically complex cases. Attendance rates at ethics and nonethics noon conferences were also compared as matched pairs. All statistical analyses were performed using IBM (Armonk, NY) SPSS Statistics 22.0. For all comparisons, a $p$ value of $<0.05$ was taken to indicate statistical significance.

RESULTS Over the course of the first 9 months of the year, we ran 1 simulation session and 12 bioethics case discussions. Each case was matched to ethics topics drawn from the AAN's Practical Ethics in Clinical Neurology curriculum.

Primary outcome. Residents averaged $75.8 \%$ on the precurriculum quiz on the management of ethically complex cases, and improved to an average of $86.7 \%$ on the postcurriculum quiz $(p=0.02)$. Each class of residents (postgraduate years 2, 3, and 4) improved their quiz performance (table).

Secondary outcomes. Self-assessed resident comfort in discussing ethically complex cases increased from a mean of 6.4 on a 10 -point scale precurriculum to a mean of 7.4 postcurriculum $(p=0.03)$. Selfassessed resident comfort in managing ethically complex cases trended toward improvement from an average of 6.5 on a 10-point scale precurriculum to an average of 7.0 postcurriculum $(p=0.07)$.

The average attendance for ethics discussions was $73.5 \%$, as compared to $61.7 \%$ for noon conferences on the days preceding ethics discussions $(p=0.04)$.

DISCUSSION This study formally examines the effects of implementing the AAN's recently published bioethics curriculum for neurologists. It showed an improvement in residents' ability to analyze ethically complex cases on precurriculum and postcurriculum multiple-choice tests. The study also showed an improvement in residents' self-assessed comfort in 
Table Precurriculum and postcurriculum quiz and self-assessment results

\begin{tabular}{|lll|}
\hline & Precurriculum & Postcurriculum \\
\hline Postgraduate year 2 ( $\mathbf{n}=\mathbf{7})$ & & \\
\hline Quiz scores, \% & 82.9 & 88.6 \\
\hline Comfort managing cases & 6.57 & 7.14 \\
Comfort discussing cases & 5.29 & 7.43 \\
\hline Postgraduate year 3 $(\mathbf{n}=\mathbf{9})$ & & \\
\hline Quiz scores, \% & 64.4 & 77.8 \\
\hline Comfort managing cases & 6.22 & 7.33 \\
\hline Comfort discussing cases & 7.11 & 7.56 \\
\hline Postgraduate year 4 ( $\mathbf{n}=\mathbf{8})$ & & 95.0 \\
\hline Quiz scores, \% & 82.5 & 6.63 \\
\hline Comfort managing cases & 6.63 & 7.13 \\
\hline Comfort discussing cases & 6.63 & \\
\hline Total (n = 24) & & 86.7 \\
\hline Quiz scores, \% & 75.8 & 7.04 \\
\hline Comfort managing cases & 6.46 & 7.38 \\
\hline Comfort discussing cases & 6.42 & \\
\hline
\end{tabular}

discussing such cases, although not in their comfort managing such cases. The curriculum was popular with residents, as demonstrated by the consistently above-average attendance.

The strengths of the study are its use of quantitative objective and subjective assessments of residents' skill prior to and following the ethics curriculum. The study's major limitations include the relatively small number of residents available over the course of a single year, the lack of a randomized control group, and the fact that the study was performed at only a single academic medical center.

The finding that residents' quiz scores and comfort discussing ethically complex cases improved while comfort managing such cases did not raises an important consideration: is our bioethics curriculum overly focused on analysis and discussion, providing residents insufficient practice in actual management? While we incorporated role play into our curriculum in an effort to give residents an opportunity to practice case management skills under realistic conditions, this was a small component of the curriculum in its current form.

Future directions. This study provides a basis for a randomized controlled trial of the AAN's ethics curriculum, to determine whether our observed benefits are the result of the formal curriculum rather than ordinary clinical experience. These studies would ideally be performed across multiple training programs to assure generalizability of the results. Because our current results suggest that more attention is needed to manage ethically complex cases, we plan to incorporate into the curriculum 3 or 4 additional case simulations focusing on family discussions regarding goals of care, life support, and patients' religious and cultural values. These simulations and the case discussions should be aligned with specific educational milestones in keeping with the latest recommendations of the Outcome Project of the ACGME and the American Board of Medical Specialties. ${ }^{10}$

\section{AUTHOR CONTRIBUTIONS}

Benjamin Tolchin: drafting/revising the manuscript, study concept or design, analysis or interpretation of data, accepts responsibility for conduct of research and final approval, acquisition of data, statistical analysis. Joshua Z. Willey: drafting/revising the manuscript, study concept or design, analysis or interpretation of data, accepts responsibility for conduct of research and final approval, study supervision. Kenneth Prager: drafting/revising the manuscript, study concept or design, accepts responsibility for conduct of research and final approval.

\section{STUDY FUNDING}

No targeted funding reported.

\section{DISCLOSURE}

B. Tolchin reports no disclosures relevant to the manuscript. J. Willey receives K23 funding from the NIH/National Institute of Neurological Disorders and Stroke for an unrelated project and reports no other disclosures. K. Prager reports no disclosures relevant to the manuscript. Go to Neurology.org for full disclosures.

\section{REFERENCES}

1. Hafferty F, Franks R. The hidden curriculum, ethics teaching, and the structure of medical education. Acad Med 1994;69:861-871.

2. Waz W, Henkind J. The adequacy of medical ethics education in a pediatrics training program. Acad Med 1995; 70:1041-1043

3. Bernat J. Restoring medical professionalism. Neurology 2012;79:820-827.

4. Sukol R. Teaching ethical thinking and behavior to medical students. JAMA 1995;273:1388-1389.

5. American Medical Association, Medical Ethics Group. Graduate Medical Education Directory 1996-1997. Chicago: American Medical Association; 1996.

6. Bernat J, Beresford HR, Cranford RE, et al. Ethical Dimensions of Neurologic Practice. Minneapolis: American Academy of Neurology; 2000.

7. Schuh LA, Burdette DE. Initiation of an effective neurology resident ethics curriculum. Neurology 2004;62: 1897-1898.

8. Williams M, McGuire D, Rizzo M, ed. Practical Ethics in Clinical Neurology: A Case-based Learning Approach. Philadelphia: Wolters Kluwer Health; 2012.

9. Baile WF, Buckerman R, Lenzi R, Glober G, Beale EA, Kudelka AP. SPIKES: a six-step protocol for delivering bad news: application to the patient with cancer. Oncologist 2000;5:302-311.

10. Swing SR, Beeson MS, Carraccio C, et al. Educational milestone development in the first 7 specialties to enter the next accreditation system. J Grad Med Educ 2013;5: 98-106. 


\title{
Neurology
}

\author{
Education Research: A case-based bioethics curriculum for neurology residents \\ Benjamin Tolchin, Joshua Z. Willey and Kenneth Prager \\ Neurology 2015;84;e91-e93 \\ DOI 10.1212/WNL.0000000000001412
}

\section{This information is current as of March 30, 2015}

\section{Updated Information \& Services}

References

Citations

Subspecialty Collections

Permissions \& Licensing

Reprints including high resolution figures, can be found at: http://n.neurology.org/content/84/13/e91.full

This article cites 7 articles, 3 of which you can access for free at: http://n.neurology.org/content/84/13/e91.full\#ref-list-1

This article has been cited by 1 HighWire-hosted articles: http://n.neurology.org/content/84/13/e91.full\#\#otherarticles

This article, along with others on similar topics, appears in the following collection(s):

\section{All Education}

http://n.neurology.org/cgi/collection/all_education

All Ethics in Neurology/Legal issues

http://n.neurology.org/cgi/collection/all_ethics_in_neurology_legal_iss ues

\section{Methods of education}

http://n.neurology.org/cgi/collection/methods_of_education

\section{Palliative care}

http://n.neurology.org/cgi/collection/palliative_care

Professional conduct and ethics

http://n.neurology.org/cgi/collection/professional_conduct_and_ethics

Information about reproducing this article in parts (figures,tables) or in its entirety can be found online at:

http://www.neurology.org/about/about_the_journal\#permissions

Information about ordering reprints can be found online:

http://n.neurology.org/subscribers/advertise

Neurology ${ }^{\circledR}$ is the official journal of the American Academy of Neurology. Published continuously since 1951, it is now a weekly with 48 issues per year. Copyright () 2015 American Academy of Neurology. All rights reserved. Print ISSN: 0028-3878. Online ISSN: 1526-632X.

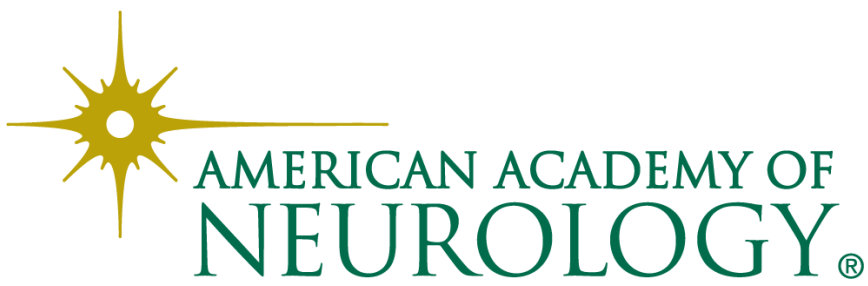

